\title{
Creating connections: Strategies to improve adolescent boys' access and engagement with school-based health services
}

Key words: Adolescent boys, School Health Services, Access, Engagement, Communication, Connections

\section{Introduction}

Adolescents, particularly boys, commonly engage in risk-taking behaviors that may compromise their short- and long-term health and wellbeing. They face issues such as substance use, sexual behaviors, eating disorders, delinquency, violence, stress and depression (Barnes, Walsh, Courtney \& Dowd 2004b; Quine et al 2003), the latter being a significant cause of morbidity and mortality during adolescence (Atkinson, Schattner \& Margolis 2003). Viner and Barker (2005) highlighted the strong continuities between adolescent and adult health, and asserted adolescence is a critical period for engaging young people in health promoting behaviors. A number of Australian states have initiated secondary school-based youth health nurse services to address adolescent health.

The School Based Youth Health Nurse (SBYHN) service in Queensland, Australia, is a program aiming to promote adolescents' health and wellbeing and provide timely support (Barnes, Courtney, Pratt \& Walsh 2004a). Guided by a Health Promoting Schools approach (Queensland Health 2006), the SBYHN program facilitates whole of school health and wellbeing through: health promotion activities; health education; resource provision; support for health-focused curriculum development; formation of partnerships between the school and the broader 
community; and individual health consultations with students, parents and members of the school community.

Timely access to primary health care services is important for adolescent boys who have a greater propensity to engage in risk-taking behaviors, may be less well equipped to make informed health decisions (Courtenay 2003), and be less likely to exhibit conventional signs of mental ill health, because depressive symptoms may be masked by disruptive behaviours and substance abuse (Wignall 2006). Despite important health needs, boys under-utilize the SBYHN service. This is consistent with findings in other settings indicating that adolescent boys access formal help services much less frequently than girls and have more negative attitudes toward help-seeking (Booth et al 2004; Schonert-Reichl \& Muller 2006).

Boys will often project a tough and confident image to adhere to conventional norms of masculinity, however beneath this façade many may be lonely, sad and confused (Martino \& Pallotta-Chiarolli 2001; Pollack 1999). Lloyd (2002) interviewed 25 young men aged 15 to 22 years who expressed a common belief that showing feelings was embarrassing, and displaying emotion would lead to vulnerability and a lack of control. While some boys were willing to talk about their feelings, many found it difficult. Kraemer (2000) argued that gender socialisation results in boys having difficulty discerning how they feel, making them less likely to ask for help when they need it. Rickwood, Deane, Wilson and Ciarrochi (2005) investigated factors affecting help-seeking amongst young people for mental health problems and reported that emotional competence levels were significantly related to help-seeking behaviour, and that boys in particular have difficulty recognising their emotional state, and may have limited ability to express how they are feeling. 
Although studies have explored specific barriers to adolescent boys' help seeking and their access to primary health care services (Bernard et al 2004; Booth et al 2004; Freak, Barley \& Kent 2007; Gleeson, Robinson \& Neal,2002; Kang et al 2006; Kay et al 2006; Kuhl, Jarkon-Horlick \& Morrisey 1997; Lindsay \& Kalafat 1998; Rickwood et al 2005; Timlin-Scalera, Ponterotto, Blumberg \& Jackson 2003; Wilson \& Deane 2001), few have explored approaches to overcoming these barriers. Freak et al (2007) called on researchers to explore how professional helpers could work more effectively with young people. This study answers this call by investigating the processes and strategies used by nurses to address barriers to boys' access to and engagement with school-based youth health services.

\section{Aim of study}

The aim of this study was to explore the processes and strategies used to promote boys' access to and engagement with SBYHN services.

\section{Methodology}

Grounded theory methods use systematic procedures to discover underlying social forces that shape human behavior (in this instance facilitating access to services), and enable theory development about social processes that may inform practice change (Strauss \& Corbin 1990). This study was based on the constructivist approach to grounded theory, which has its roots in relativism and subjectivism, and leads to the development of contextually-based theory co-created by the researcher and participants (Annells 1996b; Charmaz 2000; Mills, Bonner \& Francis 2006). Charmaz (2000) claimed the advantages of a constructivist approach to grounded theory include flexibility and increased interpretive understanding due to the focus on meaning attributed by participants. This 
approach allowed investigation of how school-based youth health nurses construct an environment that facilitates boy's access to services.

Following ethics approval from Griffith University and relevant schools and health services, interviews were conducted with ten SBYHNs working in 10 different state high schools in south-east Queensland, Australia. An interview guide consisting of open-ended questions was used. In-depth interviews using a conversational approach were used to explore perceived barriers and facilitators of boys' access to SBYHN services, as well as strategies SBYHNs used to facilitate access and engagement, and overcome barriers. Probing questions were used to explore areas raised by participants. The interview guide was revised as data collection progressed to explore themes emerging from analysis. The final interview guide (Table 1) developed from this iterative theoretical sampling process was reflective of the emerging themes.

[Insert Table 1 here]

Initially, purposive sampling was employed to select SBYHN participants in both rural and metropolitan schools in southern Queensland, from both genders, and with a range of ages and levels of experience. New research sites and SBYHN participants were selected utilizing a theoretical sampling process based upon the emerging categories, to enable comparison with data already collected and develop a deeper conceptual understanding of the phenomena under study (Glaser \& Strauss 1967). For example, data were explored for nurses who reported high numbers of boys accessing their services as well as those reporting low numbers. The final sample comprised seven female and three male nurses ranging in age from 30 to 50 years (Table 2). 
The interviews lasted an average of 45 minutes and were audio-taped and transcribed verbatim. A journal was maintained by the researcher to record thoughts, impressions and emotional reactions so as to assist with comprehending the generated data. Transcribed data were analyzed using the constant comparative method of data analysis (Strauss \& Corbin 1990), with saturation of concepts achieved.

[Insert Table 2 here]

Constant comparison involved open coding, which entailed examination of each line of data to discern the main concepts they pertained to, as well as the actions or events contained within these concepts. This resulted in the identification of over 100 concepts or open codes surrounding factors that either facilitated or impeded male adolescent students' access to the school nursing service and their engagement with the SBYHN.

Codes were compared with each other and collapsed into categories, or groups of concepts with similar characteristics. The dimensions of these categories were then identified and reassembled through a process of axial coding, where categories were linked to subcategories (Charmaz 2000). An example of this process was the creation of the category Developing trust through grouping the concepts: Actively supporting the process of referring on, Being transparent in dealings, Ensuring privacy, Expounding confidentiality, Respecting boundaries, Setting the pace of disclosure and Testing the waters. This process of coding and categorising continued until no new information was revealed through data collection and the categories were rich and complete (Strauss \& Corbin 1990). 
The dimensions of these categories were then identified and reassembled through a process of axial coding, or linking categories to subcategories (Charmaz 2000). This resulted in the conceptualization of the three main categories or strategies relating to the phenomenon under study: Facilitating communication, Mediating contextual issues, and Bridging culture, as well as the intervening conditions that served to facilitate or impede boys' access to the school based nursing service and their engagement with the SBYHN. These processes explained how the participants interacted within the school environment and with adolescent boys to address these factors and promote engagement and access.

A process of selective coding was then used to identify the core category, or the central process around which other categories were arranged in a systematic and supportive relationship (Strauss \& Corbin 1990). This occurred by questioning why certain SBYHNs were able to attract significant numbers of boys to engage with their service despite barriers within their contextual setting. The strategies used to connect with boys in meaningful and relevant ways, both collectively and individually, were reviewed and thus the storyline and core category 'Creating connections to promote access and engagement' was conceptualized.

\section{Results}

The core category, Creating Connections, was determined to consist of three processes: Facilitating communication, Mediating contextual issues and Bridging culture. These processes are represented in the Creating Connections model (Figure 1).

[Insert Figure 1 here] 
The study highlighted that participants thought the predominant reason boys do not readily seek help from their health services is a pervading socio-cultural context, generally in society and more specifically within individual school settings. They indicated that boys tend to equate help-seeking with a perception of weakness, and admitting a need for support in a school setting makes boys vulnerable to peer group bullying and ostracism. For example, Kristy ${ }^{1}$ reflected that:

It's the taunting by their mates, and to be seen as less of a man type stigma attached to that. 'Oh, do you have problems what's wrong?'.

Boys' utilisation of the SBYHN service was further influenced by intervening conditions which existed on continua, having either a positive or a negative influence. Participants considered the culture of individual schools was particularly influential, either reinforcing boys' social conditioning to equate help-seeking with vulnerability, or challenging this perspective to support and encourage boys' helpseeking behaviors. Factors identified by participants that contributed to creating and maintaining a school's ethos included: level of awareness of connection between students' learning ability and behavior with biopsychosocial health and wellbeing; level of understanding of available support; extent to which boys' helpseeking behaviors were normalized through appropriate curriculum inclusion; the process for facilitating and managing referrals to the SBYHN; and whether or not referrals to the SBYHN were associated with disciplinary action.

Participants considered that recognition of the nurse and familiarity with the service were primary considerations in students' willingness to access services. The

Pseudonyms are used to protect participants' identity. All participants have been given female names to protect the identity of male participants. 
SBYHN's involvement in day-to-day school activities, the level of acceptance of the SBYHN and the level of understanding and interpretation of his/her role within the school by teachers and administrative staff directly influenced the types and frequencies of student referrals, as well as boys' willingness to access the service. Many SBYHNs were concerned their service was often only understood in terms of traditional nursing roles related to physical ailments and injuries, rather than the broader health promotion services actually offered. Others, however, felt that traditional perceptions of nursing services created access opportunities, as boys were more comfortable discussing their physical health than emotional and psychosocial health matters.

Sarah: They see that sometimes it is easy to talk about your physical problem, like if you've got something wrong with you, and from there if there any other issues that you can help with then I think that's an 'in'.

How a nurse's particular socio-demographic characteristics (such as gender and age) influenced his/her ability to attract and engage adolescent boys varied. Both male and female SBYHN participants of similar ages reported vastly different experiences in their ability to engage boys with their service.

Students' knowledge about where the SBYHN office was located, how close it was to main thoroughfares, whether it was positioned in a potentially stigmatizing area of the school, and the level of privacy afforded to students when negotiating access to the SBYHN was perceived to have varying degrees of influence on boys' willingness to utilize the service. For example, some participants felt that locating the SBYHN's office in the administrative block inhibited boys from accessing their service, because appointments negotiated through reception staff associated them 
with school authority figures. Other participants thought co-location facilitated access because it created opportunities to approach and build a rapport with boys attending school administration for other reasons. Once attending the SBYHN service, participants thought it was important to create a conducive environment, an office conveying a message that the SBYHN was "... a different adult... not a disciplinarian... here in an unconditional context" (Nancy).

\section{Creating Connections}

The challenge for SBYHNs was to overcome the social and physical barriers to boys' access to their health services. The Creating Connections model developed from these data is a multi-faceted approach to achieving connections, consisting of three processes: Facilitating communication, Mediating contextual issues and Bridging culture. Strategies from these processes built on and synergized with each other, creating an integrated process for facilitating adolescent boys' access to health services and creating connections.

\section{Facilitating communication}

To Facilitate communication SBYHNs interacted with boys, both individually and collectively, to engage with them, promote the service, and maintain their profile within the school. To communicate effectively with individual boys during a consultation, SBYHNs worked to increase boys' comfort during the consultation. Building rapport encompassed communication strategies to maximize engagement, connect with boys' interests, to demonstrate value of him as a person, and use a boy's interests as a point of reference throughout the consultation:

Sarah: ... usually when I talk with them [boys] it sometimes can be in a round-about way, so that we, I guess, try to identify things that interest them ... Making them feel more comfortable 
about at least I am interested in them - I'm not interested in just their behaviours or some of their issues.

Participants used games and activities (such as slinkies and balls) together with sensitive and open body language to build rapport and deflect a consultation's intensity. Male-oriented analogies were used to promote understanding of health topics. They thought it was important to maintain a professional approach, genuineness and empathy during consultations, while still injecting a sense of humor.

Heidi: I really think they want to have a connection with that person first before they'll talk about anything that really matters (...) .the way you come across makes a big difference ...if you come across genuine ...that makes a big difference.

Participants felt that a sense of trust was paramount. SBYHNs explained that the service is confidential, and provided relevant examples of when confidentiality needs to be breached. They explained how they allowed boys to set the pace of disclosure, which they felt instills a sense of trust and rapport. Jan explained: And then, you know, it just might be slowly (...) sure enough, after a few visits like, that they'll actually plonk themselves down and just start to talk about issues.

Participants identified that being transparent about actions that will be taken as a result of a consultation maintains a sense of trust and connection. Engagement is facilitated by providing non-judgmental reassurance and creating a sense of freedom in decision-making about their situation. 
How a SBYHN communicates collectively with boys in the school can increase boys' familiarity with them, promote the nursing service and have a significant impact on perceptions and access. Traditional means of role promotion, such as newsletter items, student notices and brief announcements at assemblies, were not considered an effective way of communicating with boys. Participants raised their profile and promoted their role by maintaining visibility in the school grounds, projecting a happy disposition, initiating interactions, and providing opportunities to build a sense of familiarity and trust.

Jan: I like to be out. I like to wander around during their lunch times saying hello... just being really quite open. I think being quite opportunistic with boys [is important], and that [it] might be not just thinking, not just being in your room where you wait for them to come.

SBYHNs promoted services by inviting small groups of boys to the health service to familiarize them with the environment, explain how to negotiate access, and to make them feel more comfortable with both the environment and them as a service provider. To promote their service, they encouraged senior students to promote the SBYHN role to younger boys and to role-model help-seeking behaviors. Additionally, SBYHNs found that maintaining a high profile with teachers facilitated appropriate referral of boys to the nursing service, enabled greater access to classrooms and increased students' familiarity with the SBYHN and their role. SBYHNs promoted their role by placing signs around the school and/or on students' computer desktops displaying the SBYHNs' photograph and a description of the issues about which they could be consulted. 
Potential strategies for alternative ways of accessing the SBYHN, such as e-mail or a text service, were explored in this study. Electronic communication has been shown to be an effective way of decreasing the stigma boys attach to help-seeking behaviors and reducing the potential risk of embarrassment and rejection by their peers if seen physically accessing a support service (Borzekowski \& Rickert 2001; Education Queensland 2006; Gould et al 2002; Nicholas, Oliver, Lee \& O’Brien 2004, Utting 2004; Viner \& Barker 2005). Some SBYHN participants were concerned that anonymous electronic communication would create difficulties with confidentiality and they would not be able to follow-up students at risk of harm.

Sonia: I would be concerned, I think, if a student was to send me an email and I couldn't actually identify the student or I couldn't then go and follow up with the student in person if I identified that there may be a real risk to their health or wellbeing.

Some SBYHN participants were concerned about accessing e-mails in a timely fashion due to competing work demands, and difficulties associated with reliable access to computer networks. One participant was emphatic she would not use electronic communication. She thought that boys convey a lot of what they are saying through their use of body language, and that it would be very difficult to establish a therapeutic relationship if not communicating with them in person.

Tania: I rely too much on non-verbals ... I don't think it would work, because as I said boys are doers, and that's more talking.

\section{Mediating contextual issues}

Contextual issues relate not only to difficulties associated with providing a nursing service within a school setting, but also to boys' social context, particularly gender socialization and developmental considerations. 
Although SBYHNs cannot always control their physical location and resources, participants noted that environmental structures maximizing privacy can facilitate help-seeking behaviors by adolescent boys in the school setting. An office with direct, though screened, access enables boys to see the SBYHN without needing to negotiate an appointment via a third party, or sit in a waiting area where they can potentially be viewed by their peers.

Sarah: ... if you have to negotiate your way through, someone is probably going to see you somewhere, and that means... you've got a problem or there's something wrong with you, and they just don't want to come.

Negotiating for an office that is not located in a potentially stigmatizing area of the school, such as a special education unit, was also viewed as beneficial. Maintaining boys' privacy once in the SBYHN's office is another consideration, although screening the area needs to be balanced with a boy's comfort with being in an enclosed environment.

Participants described ways they strived to create a non-clinical office environment that maximizes boys' comfort and their ability to relax and relate with the SBYHN. To create a 'safe' and informal atmosphere that facilitates boys' engagement the participants used comfortable chairs or beanbags arranged to minimize a confrontational style of interaction, colorful posters, and novelty items appealing to boys. When engaging boys in an office environment was difficult, an alternative venue or an outdoor activity was used, although SBYHNs considered factors such as confidentiality surrounding their issue of concern and whether the boy was likely to experience social harm if seen accessing the health service. 
Participants identified that adolescent boys can have minimal insight into their issues and how they feel in response to their situations. Through appropriate use of speech and language to facilitate boys' understanding, SBYHNs prompted them to work through their issues while encouraging them to recognize their feelings and providing a safe atmosphere to express emotions. Providing assurance that their issues and emotions are 'normal' for their developmental stage was also considered helpful. Acknowledging that boys may experience angry feelings and providing services in a non-judgmental way provided a safe place to express and vent emotion, further facilitating access.

Heidi: ...the relaxation and the letting go side of things - helping to control your reactions, because a lot of boys have got a lot of anger, so the minute something happens [snaps her fingers] they fire up. So if you can try and help them to calm that down, that makes a big difference.

Reframing the nursing service to avoid an immediate assumption that boys who attend are experiencing significant problems, was seen to be a key factor in facilitating boys' access to the SBYHN. Sporadic, large-scale health promotion activities were seen by some to have limited effect in attracting boys to the service. As a way of breaking down the stigma of attending the health service, some participants reoriented the focus of their service to encompass more regular healthpromoting activities, for example, by providing physical activities for boys during break times or by linking with health professionals dealing with sports-related matters. Encouraging regular involvement of senior male students with organization and delivery of health-promoting activities was seen as an ideal way of modeling acceptance of using SBYHN services, and reframing how the service was viewed. 
Sarah: I think that the senior boys feel they have rights and that they are individuals and they can seek help. Whereas sometimes the younger students are still... indoctrinated into... do as you're told and don't step outside the norm in the school... they [the older boys] can encourage the young boys to come.

Another way of promoting the service was to appeal to adolescent boys' increasing desire for independence by highlighting that they can access information or support from the SBYHN without needing parental permission.

A contextual issue that concerned participants was how education and behavior management concerns are managed within the school. They explained that when boys experiencing problems are referred to senior male school administrative staff, they may not assess them holistically or consider health issues. As a consequence, an opportunity for professional input from the SBYHN is lost. Other issues arising when there are behavior management concerns include: case managers limiting access to support personnel in an effort to prevent over-utilizing support services, and boys' reluctance to divulge their information to 'yet another' adult. To address these concerns, participants strived to develop good working relationships with school administrative staff and other members of the support services team in order to address and advocate for boys' access to health services in the school.

Sarah: a lot of it tends to be around behaviour and discipline ... [s]o, not a lot of issues around, probably their health and wellbeing .. [w]e do try to work together if they do have those issues, and I do try to advocate for them, but sometimes it's really difficult in schools. 


\section{Bridging culture}

To effectively create connections, SBYHNs needed to bridge societal cultural factors that significantly influence adolescent boys' help-seeking behaviors, including the specific culture of the school and related to individual boys themselves.

To facilitate appropriate referrals to the school-based health service, participants worked to promote school staff's understanding of the links between students' behavior and learning abilities with physical and psychosocial health status. They identified that appropriate referral ensures timely access to support services, and also fosters an ethos that supports and encourages help-seeking behaviors.

Sonia: In this school there seems to be a little bit more of helpseeking promotion. There seems to be a little bit more sensitivity regarding seeking help here. For example if you have an issue with your behavior, "do you think you would benefit by talking to the counselor or talking to the nurse or talking to the chaplain?" "Is there something that they could help you with in your life that may be contributing to your behavior?"

Participants advocated for staff to actively and sensitively support boys through the referral process to facilitate attendance, which was particularly important for common restrictions surrounding referral follow-up, such as not being permitted to call students out of class to attend an appointment. 
To avoid misconceptions of the SBYHN's role within the school, participants worked to ensure referrals to the SBYHN were not associated with disciplinary action:

Sarah: I think a lot of them think it's a disciplinary thing when they come to see me ... "Well, you're in trouble for this, this and this behavior so off you go, you have to see the nurse", because it's a health-related thing.

SBYHNs worked to educate teaching and administration staff to make sure boys' access was not hindered due to concerns about class avoidance, particularly for boys with an ongoing health issue. Further ways SBYHNs enhanced the ethos of the school to be more supportive of help-seeking behaviors were to advocate for appropriate curriculum inclusion that challenges traditional norms of behavior for boys, and provide boys with skills to increase social competence. They encouraged male staff to actively role-model supportive behaviors for boys to help break down gender stereotypes and normalize help-seeking behaviors.

Nancy: I think it's about teaching that it's OK to actually talk to appropriate people at the right time about issues that are happening ... breaking down those sorts of stereotypes.

\section{Discussion}

The findings from this study complement those of previous studies on adolescent help-seeking reported in the literature, particularly barriers to communicating with adolescent boys (Atkinson et al 2003; Gleeson et al 2002; Timlin-Scalera et al 2003), and characteristics of adults preferred by adolescents seeking help in a school setting (Lindsay \& Kalafat 1998). Timlin-Scalera et al (2003) also highlighted 
the primary focus for boys was to be seen to be 'fitting in' to their social context, and discussed how help seeking from a professional was perceived to be a sign of weakness that resulted in loss of status and respect amongst their peers. The authors also described anger as the dominant and most socially-accepted emotion for adolescent boys to display, that often resulted in their real needs being overlooked (Timlin-Scalera et al , 2003).

Although gender socialization issues and contextual factors have a major influence on boys' willingness to seek help within the school setting, this present study highlighted how these factors do not preclude the SBYHN from effecting change within his/her school to facilitate greater help-seeking behaviors by boys. This is predominantly achieved by the SBYHN reframing his/her service provision to reduce the stigma attached to boys attending the health service; maintaining a high profile within the school to promote a sense of familiarity amongst boys; advocating for implementation of programs to promote greater acceptance of help-seeking behaviors; and promoting a more supportive school ethos. Specific approaches that SBYHNs may take to achieve these outcomes have been delineated through this study, and an awareness of these strategies may equip SBYHNs to work strategically to facilitate boys' access to the nursing service and engage with them in an effective manner.

\section{Limitations of the study}

This study was undertaken in two health districts in South East Queensland. Other contextual factors may have been evident had this study have been undertaken in other areas, particularly in more isolated regions or where certain ethnic and racial 
cultures are predominantly represented. As a consequence, it is difficult to generalise the results of this study to all secondary schools. However, despite these limitations, categories were saturated and findings provide conceptual understanding that may inform the provision of health services for boys.

A further limitation is that this study focused on the nurses' perspectives and strategies. Future research could explore the acceptability of strategies to support boy's help-seeking from the perspectives of adolescent boys as consumers of these primary health care services. However, understanding nurses' processes and strategies provides a first step towards developing better approaches for promoting boys' use of health services in schools.

\section{Conclusion}

A theoretical understanding of the processes involved in creating connections with adolescent boys developed through this study may inform relevant program management and practice guidelines for nurses working in school settings, particularly to promote greater collaboration between school nurses and school personnel to facilitate help seeking by boys and garner support for appropriate resourcing. Furthermore, greater awareness of the specific interpersonal skills required by school nurses to communicate effectively with boys, manage their profile within the school, and negotiate effectively with teaching and administrative staff to foster a supportive school ethos may also assist school nurse recruitment, orientation and staff training endeavors.

Implementing strategies to promote greater access for adolescent boys to school nurse services creates a unique opportunity for boys to positively interact with a 
health professional in a familiar setting, which may increase their propensity to engage in help-seeking behaviors from other health service providers beyond the school setting. This has the potential to change perceptions of socially accepted norms of behavior for adolescent boys that may, within a supportive school environment, continue on to future generations of boys. With the appropriate knowledge, skills and support, school nurses are well equipped to effect this change and significantly impact on boys' help-seeking behaviors. 


\section{References}

Annells M (1996a) Grounded theory method, part II: Options for users of the method, Nursing Inquiry 4: 176-180.

Annells M (1996b) Grounded theory method, part I: Within the five moments of qualitative research, Nursing Inquiry 4: 120-129.

Atkinson K, Schattner P and Margolis S (2003) Rural secondary school students living in a small community: Their attitudes, beliefs and perceptions towards general practice, The Australian Journal of Rural Health 11(2): 73-80.

Barnes M, Courtney M, Pratt J and Walsh A (2004a) School-Based Youth Health Nurses: Roles, responsibilities, challenges, and rewards, Public Health Nursing 21(4):316-322.

Barnes M, Walsh A, Courtney M and Dowd T (2004b) School-Based Youth Health Nurses' role in assisting young people access health services in provincial, rural and remote areas of Queensland, Australia, Rural and Remote Health, 4:279, accessed at http://rrh.deakin.edu.au on 5 December 2008.

Bernard D, Quine S, Kang M, Alperstein G, Usherwood T, Bennett D and Booth M (2004) Access to primary health care for Australian adolescents: How congruent are the perspectives of health service providers and young people, and does it matter? Australian and New Zealand Journal of Public Health 28(5):487-492.

Booth M, Bernard D, Quine S, Kang M, Usherwood T, Alperstein G and Bennett D (2004) Access to health care among Australian adolescents: Young people's perspectives and their sociodemographic distribution, Journal of Adolescent Health 34(1):97-103.

Borzekowski D and Rickert V (2001) Adolescent cybersurfing for health information, Archives of Pediatrics and Adolescent Medicine 155(7):813-817.

Charmaz K (2000). Grounded theory: Objectivist and constructivist methods, in N Denzin \& $Y$ Lincoln (eds), Handbook of qualitative research $2^{\text {nd }}$ edn, Sage Publications, Inc,. Thousand Oaks, California.

Courtenay W (2003) Key determinants of the health and the well-being of men and boys, International Journal of Men's Health 2(1):1-27.

Education Queensland (2006) Education views, The learning place: New ways of learning. Focus on counseling goes online, accessed at http://education.qld.gov.au/learningplace/pdfs/edviews/apr21-06.pdf on 10 December 2008.

Freak H, Barley V and Kent, G (2007) Adolescents' views of helping professionals: A review of the literature, Journal of Adolescence 30:639-653.

Glaser B and Strauss A (1967) The discovery of grounded theory: Strategies for qualitative research, Aldine de Gruyter, Hawthorne NY.

Gleeson C, Robinson M and Neal R (2002) A review of teenagers' perceived needs and access to primary health care: Implications for health services, Primary Health Care Research and Development 3(3):184-193.

Gould M, Munfakh, J, Lubell, K, Kleinman, M and Parker S (2002) Seeking help from the internet during adolescence, Journal of the American Academy of Child and Adolescent Psychiatry 41(10):1182-1189. 
Kang M, Bernard D, Booth M, Quine S, Alperstein G, Usherwood R and Bennett D (2003) Access to primary health care for Australian young people: Service provider perspectives, British Journal of General Practice 53:947-953.

Kay C, Morgan D, Tripp J, Davies C and Sykes S (2006) To what extent are school drop-in clinics meeting pupils' self-identified health concerns? Health Education Journal 65(3): 236251.

Kraemer S (2000) The fragile male, British Medical Journal 321(7276):1609-1612.

Kuhl J, Jarkon-Horlick L and Morrisey R (1997) Measuring barriers to help-seeking behaviours in adolescents, Journal of Youth and Adolescence 26:637-651.

Lindsay C and Kalafat J (1998) Adolescents' views of preferred helper characteristics and barriers to seeking help from school-based adults, Journal of Educational and Psychological Consultation 9:171-193.

Lloyd T (2002) Soldier it! Young men and suicide. Men's health forum, accessed at http://www.malehealth.co.uk/uploaded files/mhfsuicideauditfinal.pdf on 9 December, 2008.

Martinelli W and Pallott-Chiarolli M (2001) Boys' stuff: Boys talking about what matters, Allen \& Unwin, Crows Nest NSW.

Mills J, Bonner A and Francis K (2006) Adopting a construvist approach to grounded theory: Implications for research design, International Journal of Nursing Practice 12:8-13.

Nicholas J, Oliver K, Lee K and O'Brien M (2004) Help-seeking behaviour and the internet: An investigation among Australian adolescents, Australian e-Journal for the Advancement of Mental Health 3(1), accessed at http://www.ausinet.com/journal/vol3iss1/nicholas.pdfD on 9 December 9, 2008.

Pollack W (1999) Real boys: Rescuing our sons from the myths of boyhood, Henry Holt \& Co, New York.

Queensland Health (2006) Memorandum of Understanding between Queensland Health and Education Queensland and Program Management Guidelines: School Based Youth Health Nurse Program. Queensland Health, Brisbane.

Quine S, Bernard D, Booth M, Kang M, Usherwood T, Alperstein G and Bennett D (2003) Health and access issues among Australian adolescents: A rural-urban comparison, Rural and Remote Health 3 (on-line): 245, accessed at http://rrh.deakin.edu.au on 8 January 2009.

Rickwood D, Deane F, Wilson C and Ciarrochi J (2005) Young people's help-seeking for mental health problems, Australian e-Journal for the Advancement of Mental Health 4(3), accessed at http://www. auseinet.com/journal/vol4iss3suppl/rickwood.pdf on 9 December 2008.

Schonert-Reichl K and Muller J (2006) Correlates of help-seeking in adolescence, Journal of Youth and Adolescence 25(6):705-731.

Strauss A and Corbin J (1990) Basics of qualitative research: Grounded theory procedures and techniques, Sage Publications, Newbury Park, California.

Timlin-Scalera R. Ponterotto J, Blumberg F and Jackson M (2003) A grounded theory study of help-seeking behaviours among white male high school students, Journal of Counseling Psychology 50(3):339-350.

Utting S (2004) How teenagers got the health message, Primary Health Care 14(2):12-14 
Viner R and Barker M (2005) Young people's health: The need for action, British Medical Journal 330(7496):901-903.

Wignall A (2006). Behaviour problems can hide depression, Mindmatters Plus: Information Sheet No. 8 accessed at http://mmplus.agca.com.au/documents/infosheet8.pdf on 9 December 9, 2008.

Wilson C and Deane, F (2001) Adolescent opinions about reducing help seeking barriers and increasing appropriate help engagement, Journal of Educational and Psychological Consultation 12:345-364. 
Figure 1: Creating Connections Model

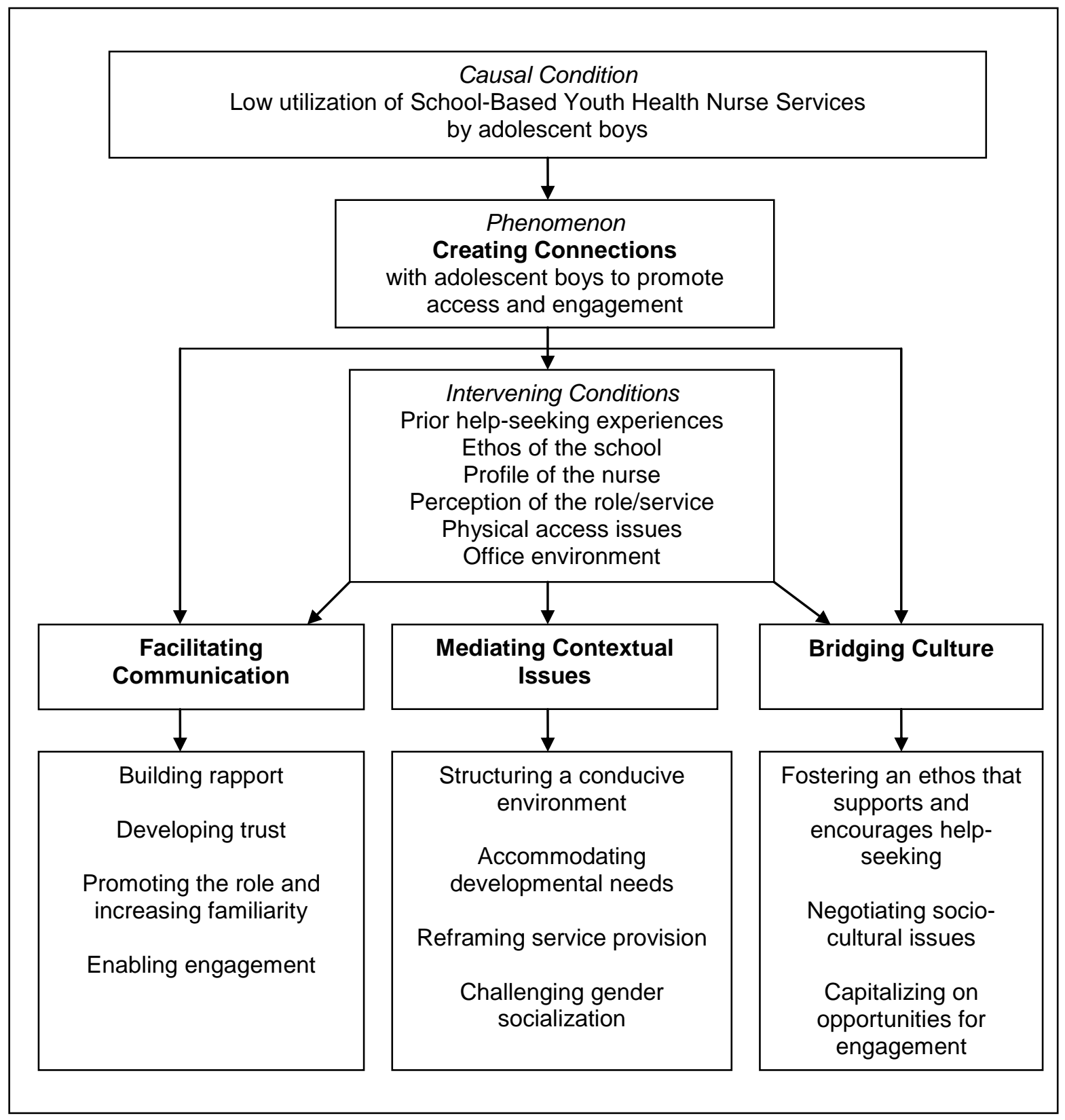




\section{Table 1: Final Interview Guide}

Inequities in gender access to the SBYHN service

- How long have you been working in the SBYHN program?

- What is the approximate ratio of boys to girls self-referring to your service?

- Describe your experience with boys who have self-referred to your service.

- Describe your experience with boys who have been referred to your service by school staff, and in particular, their willingness to self-refer on subsequent occasions.

Perceived barriers to accessing the SBYHN services

- How does the physical location or set up of your office impact on student access?

- How do you promote your service to students and staff?

- Discuss the frequency of student referrals from teachers/admin staff?

- What type of feedback have you received regarding students being reluctant to access your service due to your gender?

- Describe your own level of comfort to discuss issues of a personal nature with adolescent boys.

- What other factors do you feel may inhibit boys from accessing your service?

Facilitators to boys accessing the SBYHN services

- What strategies to facilitate communication do you use when consulting with boys?

- $\quad$ Suggest some ways to increase boys' comfort when accessing the service.

- What types of strategies do you employ to actively encourage boys to access your service?

- Suggest some ways that boys may be encouraged to self-refer to the SBYHN service.

- How well would electronic access to the SBYHN service be received by students in your school and why? 
Table 2: Demographic and descriptive data: SBYHN participants

\begin{tabular}{|l|r|}
\hline Gender of SBYHN participants & 7 Females; 3 Males \\
\hline Context & $\begin{array}{r}\text { 2 rural schools; } \\
\text { 14 metropolitan schools }\end{array}$ \\
\hline Age & 5 months - 7 years \\
\hline $\begin{array}{l}\text { Length of service with the SBYHN } \\
\text { program }\end{array}$ & 16 schools \\
\hline $\begin{array}{l}\text { No. of schools serviced by participants } \\
\text { during data collection period }\end{array}$ & \begin{tabular}{l} 
Lowest reported: Boys 0\%; Girls 100\% \\
Highest reported: Boys 60\%; Girls 40\% \\
\hline $\begin{array}{l}\text { Estimated ratio of boys to girls currently } \\
\text { self-referring to SBYHNs }\end{array}$
\end{tabular} \\
\hline
\end{tabular}

NB. Demographic data grouped to maintain confidentiality. 